\title{
FIELD EQUIVALENT FINITE GROUPS
}

\author{
by \\ Alexander Moretó and Gabriel Navarro \\ Facultat de Matemàtiques \\ Universitat de València \\ Burjassot, València 46100 \\ SPAIN \\ E-mail: alexander.moreto@uv.es,gabriel@uv.es
}




\section{INTRODUCTION}

M. Isaacs has given the following definition: two finite groups $X$ and $Y$ are field equivalent if there is a bijection $\chi \mapsto \chi^{\prime}$ from $\operatorname{Irr}(X)$ onto $\operatorname{Irr}(Y)$ such that $\mathbb{Q}(\chi)=\mathbb{Q}\left(\chi^{\prime}\right)$ for every $\chi \in \operatorname{Irr}(X)$, where $\operatorname{Irr}(X)$ is the set of complex irreducible characters of $X$ and $\mathbb{Q}(\chi)$ is the field of values of $\chi$. In this paper, we give solution to a problem proposed by him.

THEOREM A. Suppose that $G$ is field equivalent to a cyclic group. Then $G$ is cyclic.

In general, we cannot expect much more than this. For instance, there exists a group $G$ of order 64 with 16 conjugacy classes such that all of its irreducible characters are rational valued. Hence, $G$ is field equivalent to an elementary abelian 2-group and $G$ is not abelian. Even more, there exists another group $H$ of order 32 with 11 conjugacy classes and rational valued characters. In particular, $H$ is field equivalent to the symmetric group of degree 6 .

There is an application of Theorem A: if $A$ acts coprimely on a finite group $G$, then the fields of values of the $A$-invariant irreducible characters of $G$ determine if the fixed points subgroup $\mathbf{C}_{G}(A)$ is cyclic. (See Section 4 below.)

\section{GROUPS OF ODD ORDER}

We notice that a finite group $G$ is field equivalent with a cyclic group $C$ of order $n$ if and only if

$$
\operatorname{Irr}(G)=\bigcup_{d \mid n} \operatorname{Irr}_{d}(G)
$$

where $\operatorname{Irr}_{d}(G) \cap \operatorname{Irr}_{e}(G)=\emptyset$ if $d \neq e,\left|\operatorname{Irr}_{d}(G)\right|=\varphi(d)$, and if $\psi \in \operatorname{Irr}_{d}(G)$, then $\mathbb{Q}(\psi)=\mathbb{Q}_{d}$, the cyclotomic field of $d$-th roots of unity. This easily follows by writing $\operatorname{Irr}_{d}(C)=\{\lambda \in$ $\operatorname{Irr}(C) \mid o(\lambda)=d\}$, and noticing that if $\lambda \in \operatorname{Irr}_{d}(C)$, then $\mathbb{Q}(\lambda)=\mathbb{Q}_{d}$. Since groups of odd order are exactly the groups with exactly one real character, we have that $|G|$ is odd if and only if $n$ is odd.

In order to use inductive arguments in groups of odd order, it is convenient to have the following weaker hypothesis.

(2.1) HYPOTHESIS. Suppose that $G$ is a finite group such that

$$
\operatorname{Irr}(G)=\bigcup_{d \in A} \operatorname{Irr}_{d}(G)
$$

where $A$ is a set of positive odd integers such that if $\psi \in \operatorname{Irr}_{d}(G)$, then $\mathbb{Q}(\psi)=\mathbb{Q}_{d}$ and $\left|\operatorname{Irr}_{d}(G)\right|=\varphi(d)$.

Our aim in this Section is to classify all finite groups satisfying Hypothesis (2.1).

Throughout this paper, we shall use an elementary fact on cyclotomic fields: if $d \leq e$ are positive integers, then $\mathbb{Q}_{d} \subseteq \mathbb{Q}_{e}$ if and only if $d$ divides $e$ or $e$ is odd and $d=2 f$, for some $f$ dividing $e$. Hence, if $e$ and $d$ are odd, then $\mathbb{Q}_{d} \subseteq \mathbb{Q}_{e}$ if and only if $d$ divides $e$ and therefore $\mathbb{Q}_{d}=\mathbb{Q}_{e}$ only if $d=e$. If a group $G$ satisfies (2.1) and $d \in A$, then notice that 
$G$ has exactly $\varphi(d)$ characters $\chi$ with $\mathbb{Q}(\chi)=\mathbb{Q}_{d}$ and all of them are Galois conjugate. In particular, if a group $G$ satisfies (2.1), then all factor groups of $G$ satisfy (2.1). Notice too that groups satisfying (2.1) are of odd order. Finally, if $\chi \in \operatorname{Irr}(G)$ is such that $\mathbb{Q}(\chi)=\mathbb{Q}_{f}$, where $f$ is odd, then $f \in A$.

(2.2) LEMMA. Suppose that $G$ is a nilpotent group satisfying (2.1). Then $G$ is cyclic.

Proof. Since $G / \Phi(G)$ satisfies (2.1), we may assume that the Sylow subgroups of $G$ are elementary abelian. Now let $p$ be a prime divisor of $|G|$ and let $\lambda \in \operatorname{Irr}(G)$ be of order $p$. Then $\mathbb{Q}(\lambda)=\mathbb{Q}_{p}$ and $G$ has exactly $p-1$ irreducible characters with field of values $\mathbb{Q}_{p}$. Hence all Sylow subgroups of $G$ are cyclic.

We shall repeatedly use the following fact.

(2.3) LEMMA. Suppose that $G$ has a normal Sylow $p$-subgroup $P$ and let $\theta \in \operatorname{Irr}(P)$. If $T$ is the stabilizer of $\theta$ in $G$ and $\hat{\theta}$ is the canonical extension of $\theta$ to $T$, then $\chi=\hat{\theta}^{G} \in \operatorname{Irr}(G)$ lies over $\theta$ and $\mathbb{Q}(\chi) \subseteq \mathbb{Q}(\theta)$.

Proof. By Corollary (8.16) of [3], there exists a unique $\hat{\theta} \in \operatorname{Irr}(T)$ extending $\theta$ such that the determinantal order of $\hat{\theta}$ is a power of $p$. In fact $o(\theta)=o(\hat{\theta})$. (This is called the canonical extension of $\theta$ to T.) Now, $\chi$ lies over $\theta$ and $\mathbb{Q}(\chi) \subseteq \mathbb{Q}(\hat{\theta})$. Since $\theta$ uniquely determines $\hat{\theta}$, it follows that $\mathbb{Q}(\theta)=\mathbb{Q}(\hat{\theta})$.

(2.4) THEOREM. Suppose that $G$ is a group satisfying (2.1). Suppose that $G$ has an elementary normal $p$-subgroup $V$ such that $G / V$ has a normal $p$-complement and a cyclic Sylow p-subgroup. If $\lambda \in \operatorname{Irr}(V)$ has order $p$, then $\left\{\lambda, \lambda^{2}, \ldots, \lambda^{p-1}\right\}$ is a complete set of representatives of $G$-orbits on $\operatorname{Irr}(V)-1_{V}$.

Proof. We may write $G / V=(K / V)(P / V)$, where $K / V \triangleleft G / V$ has $p^{\prime}$-order, $P \in \operatorname{Syl}_{p}(G)$ and $P / V$ is cyclic. Suppose that $|P / V|=p^{f}$. Since $P / V$ is isomorphic to a quotient of $G$, we have that for $e \leq f, G$ has exactly $\varphi\left(p^{e}\right)$ irreducible characters with field of values $\mathbb{Q}_{p^{e}}$, all having $K$ in its kernel.

Let $1 \neq \lambda \in \operatorname{Irr}(V)$ and let $T=I_{G}(\lambda)$ be the stabilizer of $\lambda$ in $G$. Now, by Corollary (8.16) of [3], there exists a unique $\hat{\lambda} \in \operatorname{Irr}(T \cap K)$ of order $p$ extending $\lambda$. Also, by uniqueness, we have that $\hat{\lambda}$ is $T$-invariant. In particular, if $L=\operatorname{ker}(\hat{\lambda})$, then $L \triangleleft T$. Also, $|(T \cap K) / L|=p$. Now, $T / T \cap K$ is cyclic, and therefore $\hat{\lambda}$ extends to $T$. Suppose that the cyclic group $T / T \cap K$ has order $p^{d}$. We have that $d \leq f$. If $\beta \in \operatorname{Irr}(T)$ lies over $\hat{\lambda}$, we have that $\beta$ extends $\hat{\lambda}$ and $\beta^{p^{d+1}}=1$. We have that $\mathbb{Q}\left(\beta^{G}\right) \subseteq \mathbb{Q}(\beta) \subseteq \mathbb{Q}_{p^{d+1}}$. Since $\mathbb{Q}\left(\beta^{G}\right)=\mathbb{Q}_{p^{e}}$ for some $e \leq d+1$ and $K$ is not contained in the kernel of $\beta^{G}$, necessarily $e>f$. Then $e=f+1, d=f, \mathbb{Q}\left(\beta^{G}\right)=\mathbb{Q}_{p^{f+1}}$ and $\mathbb{Q}(\beta)=\mathbb{Q}_{p^{f+1}}$. In particular, $o(\beta)=p^{f+1}$. Since $L \subseteq \operatorname{ker}(\beta)$, we deduce that $T / L$ is cyclic of order $p^{f+1}$. Now, by considering the $p^{f}$ extensions $\beta$ of $\hat{\lambda}$ to $T$, we notice that $G$ has $p^{f}$ different irreducible characters with field of values $\mathbb{Q}_{p^{f+1}}$ lying over $\lambda$.

Suppose now that $\lambda^{g}=\lambda^{s}$ for some $g \in G$ and $1<s<p$. Then $T^{g}=I_{G}\left(\lambda^{s}\right)=T$. Hence, $g \in \mathbf{N}_{G}(T)$. By the uniqueness of canonical extensions, we easily have that $\hat{\lambda}^{g}=\hat{\lambda}^{s}$ and also $\operatorname{ker}(\hat{\lambda})=\operatorname{ker}\left(\hat{\lambda}^{s}\right)=\operatorname{ker}\left(\hat{\lambda}^{g}\right)=L^{g}$. Thus $g$ also normalizes $L$. Write $T / L=\langle y L\rangle$ and notice that $y^{g^{-1}} L=y^{n} L$ for some $1 \leq n$ coprime with $p$. Now, let $\beta \in \operatorname{Irr}(T)$ be 
over $\hat{\lambda}$ and let $\chi=\beta^{G} \in \operatorname{Irr}(G)$, which we know has field of values $\mathbb{Q}_{p^{f+1}}$. Now, we have that $\beta^{g}=\beta^{n}$. Hence, $\hat{\lambda}^{g}=\hat{\lambda}^{n}=\hat{\lambda}^{s}$ and therefore $n \equiv s \bmod p$. Now, let $\sigma$ be the Galois automorphism of $\operatorname{Gal}\left(\mathbb{Q}_{|G|} / \mathbb{Q}\right)$ fixing $p^{\prime}$-roots of unity and sending each $p$-power order root of unity $\xi$ to $\xi^{n}$. Then

$$
\chi^{\sigma}=\left(\beta^{\sigma}\right)^{G}=\left(\beta^{n}\right)^{G}=\left(\beta^{g}\right)^{G}=\beta^{G}=\chi,
$$

and therefore $\sigma$ fixes $\mathbb{Q}_{p} f+1=\mathbb{Q}(\chi)$. Then $\sigma$ fixes $\mathbb{Q}_{p}$ and therefore $n \equiv 1 \bmod p$. Thus $s \equiv 1 \bmod p$, and this is impossible.

Hence, for each $1 \leq j \leq p-1$, we have at least $p^{f}$ irreducible characters of $G$ with field of values $\mathbb{Q}_{p^{f+1}}$ lying over $\lambda^{j}$. This gives rise to at least $p^{f}(p-1)=\varphi\left(p^{f+1}\right)$ irreducible characters, and we conclude that there are no more. This implies the theorem.

In what follows, we shall use a well-known fact: if $V$ is a faithful irreducible $G F(p) C$ module, where $C$ is cyclic of order $m$, then $|V|=p^{n}$, where $n$ is the order of $p$ modulo $m$.

(2.5) LEMMA. Suppose that $V$ is a faithful irreducible $G F(p) C$-module of dimension $n$, where $C$ is cyclic of order e coprime with $p$. Suppose that there exists $v \in V$ such that $\{v, 2 v, \ldots,(p-1) v\}$ is a complete set of representatives of $C$-orbits on $V-\{0\}$. Then $|C|=p^{n}-1 / p-1$ and $(p-1, e)=1$.

Proof. Our hypotheses easily imply that $\mathbf{C}_{C}(v)=\mathbf{C}_{C}(V)=1$ and therefore $\mathbf{C}_{C}(w)=1$ for all $0 \neq w \in V$. Hence, $|C|=p^{n}-1 / p-1=e$. Let $d=(p-1, e)$ and let $D$ be the subgroup of $C$ of order $d$. Now, let $W$ be a simple $D$-submodule of $V$. Then $W$ is faithful and if $|W|=p^{m}$, we know that $m$ is the order of $p$ modulo $d$. Hence $m=1$. If $1 \neq x \in D$ and $0 \neq w \in W$, we have that $w x=k w$ for some $1<k<p$. Now, $w=j v c$ for some $c \in C$ and $1 \leq j<p$, and we conclude that $v x=k v$. This is not possible.

In the proof of the following result, we use a nontrivial theorem of E. Shult, namely, if $A$ acts as automorphisms on an odd $p$-group $P$ transitively permuting the subgroups of order $p$ of $P$, then $P$ is abelian $([6])$.

(2.6) THEOREM. Suppose that $G$ is a group satifying (2.1) with Fitting length 2. Let $N$ be the smallest normal subgroup of $G$ such that $G / N$ is nilpotent. Then $G=N C$, where $C$ is cyclic, $(|N|,|C|)=1$ and $N$ is nilpotent such that all of its Sylow subgroups are non-cyclic elementary abelian and minimal normal subgroups of $G$.

Proof. By Lemma (2.2), we have that $G / N$ is cyclic. Also, by hypothesis, $1<N$ is nilpotent.

First, we want to see that $(|G / N|,|N|)=1$. Let $p$ be a common prime divisor of $|N|$ and $|G / N|$. If $K$ is the $p$-complement of $N$, by working in $G / K$ (which has Fitting length two) we may assume that $N$ is a $p$-group. Since $G / N$ is abelian, we have that $G$ has a normal Sylow $p$-subgroup $P>N$. We may write $G=P D$, where $D$ is a cyclic $p^{\prime}$-group, $[P, D] \subseteq N$ and $P / N$ is cyclic. Since $p$ divides $|G / N|$, we have that $G / N$ has a linear irreducible character of order $p$. Hence, all the $p-1$ irreducible characters $\psi$ of $G$ with $\mathbb{Q}(\psi)=\mathbb{Q}_{p}$ contain $N$ in the kernel. Suppose that $P$ is not cyclic. Then $P / \Phi(P)$ is not cyclic and therefore there exists $\lambda \in \operatorname{Irr}(P)$ linear of order $p$ with $N$ not contained in its 
kernel. By Lemma (2.3), there exists $\chi \in \operatorname{Irr}(G)$ lying over $\lambda$ with $\mathbb{Q}(\chi) \subseteq \mathbb{Q}_{p}$. Now, $\mathbb{Q}(\chi)=\mathbb{Q}_{f}$ for some odd integer $f$, and we deduce that $\mathbb{Q}(\chi)=\mathbb{Q}_{p}$. This is impossible. Therefore, $P$ is cyclic. Since $P=[P, D] \times \mathbf{C}_{P}(D)$, we conclude that $[P, D]=1$. Hence, $G$ is abelian, and this is a contradiction. We conclude that $(|G / N|,|N|)=1$.

We may write $G=N C$, where $C$ is cyclic and $(|N|,|C|)=1$. It remains to show that the Sylow subgroups of $N$ are non-cyclic elementary abelian minimal normal subgroups of $G$. Let $P \in \operatorname{Syl}_{p}(N)$ and notice that $P C$ is isomorphic to a factor group of $G$ with Fitting length two. Hence, it is no loss if we assume that $N=P$. Also, since $G / \mathbf{C}_{C}(P)$ cannot be nilpotent, we may assume that $\mathbf{C}_{C}(P)=\mathbf{C}_{C}(P / \Phi(P))=1$.

By Theorem (2.4), if $1 \neq \lambda \in \operatorname{Irr}(P / \Phi(P))$, we know that $\left\{\lambda, \lambda^{2}, \ldots, \lambda^{p-1}\right\}$ is a complete set of representatives of $C$-orbits on $\operatorname{Irr}(P / \Phi(P))-1_{P}$. Since $C$ is abelian, notice that all nontrivial irreducible characters of $P / \Phi(P)$ have the same stabilizer $T$. Now, the elements of $T \cap C$ fix every irreducible character in $P / \Phi(P)$ and we deduce that $T \cap$ $C=\mathbf{C}_{C}(P / \Phi(P))=1$ and $T=P$. In particular, we have that $\operatorname{Irr}(P / \Phi(P))$ is an irreducible faithful $C$-module. Thus, if $|P / \Phi(P)|=p^{n}$, by Lemma (2.5), we have that $|C|=p^{n}-1 / p-1=e$ with $(e, p-1)=1$. If $P / \Phi(P)=\langle\lambda\rangle$ is cyclic, then $n=1$ and $[C, P]=1$. Hence $G$ is nilpotent and this is not possible. Hence, $P$ is not cyclic.

Notice now that $G$ exactly has $p-1$ irreducible characters with field of values $\mathbb{Q}_{p}$, and these are lying over $\lambda, \lambda^{2}, \ldots, \lambda^{p-1}$, respectively, where $1 \neq \lambda \in \operatorname{Irr}(P / \Phi(P))$.

Suppose that $P / P^{\prime}$ is not elementary abelian. Hence $P^{\prime}<\Phi(P)$ and let $U / P^{\prime}=$ $\Phi\left(\Phi(P) / P^{\prime}\right)$. Now, $U \triangleleft G, P / U$ is abelian and $\exp (P / U)=p^{2}$. Now, $\Phi(P) / U \subseteq \Omega_{1}(P / U) \triangleleft$ $G / U$. Hence, $\Phi(P) / U=\Omega_{1}(P / U)$. In particular, $P / U$ is a direct product of $n$ cyclic groups of order $p^{2}$.

Suppose that $\mu \in \operatorname{Irr}(P / U)$ is one of the $p^{2 n}-p^{n}$ characters of $P / U$ of order $p^{2}$. By Lemma (2.3), there exists $\chi \in \operatorname{Irr}(G)$ over $\mu$ with $\mathbb{Q}(\chi)=\mathbb{Q}_{a} \subseteq \mathbb{Q}_{p^{2}}$ for some odd integer $a$. Now, $a$ divides $p^{2}$ and necessarily $a=p^{2}$. Hence there are exactly $\varphi\left(p^{2}\right)=p(p-1)$ irreducible characters in $G$ with field of values $\mathbb{Q}_{p^{2}}$. This implies that the $p^{2 n}-p^{n}$ characters of order $p^{2}$ lie in at most $p(p-1)$ different $C$-orbits. On the other hand, if $x \in C$ fixes $\mu$, then $x$ fixes $\mu^{p}$ and thus $x \in P$. Hence, each $C$-orbit exactly contains $\frac{p^{n}-1}{p-1}$ elements. Then

$$
p^{2 n}-p^{n} \leq p(p-1) \frac{p^{n}-1}{p-1}
$$

and $n=1$, which is not possible.

We wish to prove that $P$ is abelian. We may assume that $P^{\prime}$ is a minimal normal subgroup of $G$, and therefore elementary abelian. Also, $P^{\prime} \subseteq \mathbf{Z}(P)$. Since $P / P^{\prime}$ is a chief factor of $G$, we have that $Z=\mathbf{Z}(P)=P^{\prime}$. Now, the exponent of $P$ divides $p^{2}$. Hence, if $\theta \in \operatorname{Irr}(P), \mathbb{Q}(\theta) \subseteq \mathbb{Q}_{p^{2}}$. If $\theta \in \operatorname{Irr}(P)$ does not contain $P^{\prime}$ in its kernel, by Lemma (2.3), there exists $\chi \in \operatorname{Irr}(G)$ lying over $\theta$ such that $\mathbb{Q}(\chi) \subseteq \mathbb{Q}(\theta) \subseteq \mathbb{Q}_{p^{2}}$. Since the irreducible characters of $G$ with field of values $\mathbb{Q}_{p}$ contain $P^{\prime}$ in its kernel, we deduce that $\mathbb{Q}(\chi)=\mathbb{Q}(\theta)=\mathbb{Q}_{p^{2}}$. In particular, the exponent of $P$ is $p^{2}$. Now, since $P / Z$ is abelian, $Z$ is elementary abelian and $p$ is odd, we have that

$$
\Omega_{1}(P)=\left\langle x \in P \mid x^{p}=1\right\rangle=\left\{x \in P \mid x^{p}=1\right\}<P .
$$

We conclude that all the subgroups of order $p$ of $P$ lie inside $Z$. By coprime action, and using that $p$ is odd, it is well-known that $\mathbf{C}_{C}(Z)=\mathbf{C}_{C}(P)=1$. Hence $Z$ is a 
faithful irreducible $C$-module and therefore $|Z|=\left|P / P^{\prime}\right|=p^{n}$. Now, we claim that $C$ acts transitively on the subgroups of order $p$ of $Z$. Let $1 \neq z \in Z$ and suppose that $c \in C$ fixes $\langle z\rangle$. Then $z^{c}=z^{k}$ for some $1 \leq k<p$. Since $(e, p-1)=1$, we deduce that $z^{c}=z$. Then $c$ centralizes $\left\langle z^{u} \mid u \in C\right\rangle=Z$, and this is impossible. Therefore the stabilizer of $\langle z\rangle$ in $C$ is trivial. Since there are $p^{n}-1 / p-1=|C|$ subgroups of order $p$ in $Z$, we conclude that $C$ acts transitively on them. By Shult's theorem, this is a contradiction.

Finally, since $P$ is an irreducible $C$-module, we have that $P$ is a minimal normal subgroup of $G$.

In the next result, we use a well-known theorem of Brodkey ([1]): if a finite group $G$ has an abelian Sylow $p$-subgroup $P$, then there is $g \in G$ such that $P \cap P^{g}=\mathbf{O}_{p}(G)$.

(2.7) THEOREM. If $G$ satisfies (2.1), then the Fitting length of $G$ is at most 2.

Proof. We argue by induction on $|G|$. We may assume that $G$ has a minimal normal subgroup $V$ such that the Fitting length of $G$ is 3 and $G / V$ has Fitting length 2. We have that $V$ is an elementary abelian $p$-group.

By Theorem (2.6), we know the structure of $G / V$. We have that $G / V=(N / V)(C / V)$, where $N / V$ and $C / V$ are coprime, $C / V$ is cyclic and the Sylow subgroups of $N / V$ are non-cyclic elementary abelian. Also, $N$ is not nilpotent.

First, we prove that $p$ does not divide $|N / V|$. Suppose it does. By taking a linear character of $N / V$ of order $p$ and using Lemma (2.3), we see that there are exactly $p-1$ irreducible characters of $G$ with field of values $\mathbb{Q}_{p}$ all of them having $V$ in their kernel. Let $Q / V$ be a Sylow $p$-subgroup of $G / V$, which is normal in $G / V$. Also $Q / V$ is elementary abelian and $\Phi(Q) \subseteq V$. Hence, the exponent of $Q$ is at most $p^{2}$ and all irreducible characters of $Q$ have their values in $\mathbb{Q}_{p^{2}}$. Let $\mu \in \operatorname{Irr}(Q)$ be not containing $V$ in its kernel. By Lemma (2.3), there exists $\chi \in \operatorname{Irr}(G)$ such that $\mathbb{Q}(\chi) \subseteq \mathbb{Q}(\mu) \subseteq \mathbb{Q}_{p^{2}}$. Necessarily, $\mathbb{Q}(\chi)=\mathbb{Q}(\mu)=\mathbb{Q}_{p^{2}}$. In particular, $V=\Phi(Q)$. Now, we have that a $p$-complement $H$ of $N$ acts trivially on $Q / \Phi(Q)$. Thus $[H, Q]=1$. So $N$ is nilpotent and this is impossible.

Now, by Theorem (2.4), we have that the stabilizers of all nontrivial elements of $\operatorname{Irr}(V)$ are $G$-conjugate.

Now, $\mathbf{C}_{N}(V)=U \times V$, where $U \triangleleft G$ and $U \subseteq \mathbf{Z}(N)$. If $U>1$, by induction we have that $N / U$ is nilpotent, and therefore $N$ is nilpotent. So we may assume that $\mathbf{C}_{N}(V)=V$.

Let $q$ be a prime dividing $|N: V|$ and let $X / V \in \operatorname{Syl}_{q}(N / V)$. Hence, $X / V$ is a normal abelian Sylow $q$-subgroup of $G / V$. Let $S \in \operatorname{Syl}_{q}(X)$. By Brodkey's theorem, there exists $v \in V$ such that $S \cap S^{v}=1$. Therefore $\mathbf{C}_{S}(v)=1$. Since the actions of $S$ on $V$ and on $\operatorname{Irr}(V)$ are permutation isomorphic (by Theorem (13.24) of [3]), there exists $\lambda \in \operatorname{Irr}(V)$ such that $T \cap X=V$, where $T$ is the stabilizer of $\lambda$ in $G$. Now, $T \cap X / V$ is a Sylow $q$-subgroup of $T / V$ and we deduce that $T / V$ is a $q^{\prime}$-group. Now, if $\mu \in \operatorname{Irr}(V)$ and $I$ is its stabilizer in $G$, we deduce that $I / V$ is a $q^{\prime}$-group. In particular, $I \cap X=V$. Then $\mu^{X} \in \operatorname{Irr}(X)$ for all $1 \neq \mu \in \operatorname{Irr}(V)$ and we deduce that $\mathbf{C}_{S}(w)=1$ for all $1 \neq w \in V$. Then $X$ is a Frobenius group and $S$ is a Frobenius complement of odd order. Hence, $S$ is cyclic, and this is impossible.

\section{PROOF OF THEOREM A}


In the proof of our main result, we use the following result of Iwasaki ([4]). For the reader's convenience, we write down a proof.

(3.1) LEMMA. If $G$ has at most two real valued characters, then a Sylow 2-subgroup of $G$ is normal.

Proof. We argue by induction on $|G|$, and we may assume that $G$ is of even order. We have that $G$ has exactly two real classes. Hence, the only nontrivial real class $K$ is the class of involutions of $G$. If $x, y$ are involutions, then $x y$ is real, and therefore $x y$ is an involution. Thus $N=K \cup 1$ is a normal 2-subgroup of $G$. If $G / N$ has exactly one real character, then $G / N$ is of odd order, and we are done. Otherwise, we apply induction.

We will also use the following result of Amit and Chillag.

(3.2) THEOREM. Suppose that $G$ is a solvable group and let $\chi \in \operatorname{Irr}(G)$ with $\mathbb{Q}(\chi)=$ $\mathbb{Q}_{f}$. Then $G$ has an element of order $f$.

Proof. See Theorem (22.1) of [5].

(3.3) LEMMA. Suppose that $F=G F\left(2^{m}\right)$ and let $\sigma \in \operatorname{Gal}(F)$ be of order $q>1$ odd. Let $\Gamma$ be the semidirect product of $K=F^{\times}$with $I=\langle\sigma\rangle$. Suppose that $H \leq \Gamma$ is not cyclic and has order divisible by $2^{m}-1$. Then there exists $\psi \in \operatorname{Irr}(H)$ such that $\mathbb{Q}(\psi)$ is not a cyclotomic field.

Proof. We claim that there exists $P \in \operatorname{Syl}_{p}(K)$ such that $I$ acts Frobenius on $P$. Suppose that $m \neq 6$. Let $p$ be a Zsigmondy prime for $2^{m}-1$. (See, for instance, Theorem (6.2) of [5].) If $1 \neq \tau \in I$ has order $d \mid m$, then $\left|\mathbf{C}_{K}(\tau)\right|=2^{m / d}-1$ which is not divisible by $p$. If $P \in \operatorname{Syl}_{p}(K)$, we have that $\mathbf{C}_{P}(\tau)=1$. Thus $I$ acts Frobenius on $P$. If $m=6$, then $q=3$ and in this case we can take $P$ of order 7 .

Now, since $P$ is cyclic, we have that $q \mid p-1$ and $P$ is a normal Sylow $p$-subgroup of $\Gamma$. Hence, $P \subseteq H$, by hypothesis. Now, let $\lambda \in \operatorname{Irr}(P)$ be of order $p$. Notice that $I_{\Gamma}(\lambda)=K$ because $I_{I}(\lambda)=1$. Hence, $K \cap H$ is the stabilizer of $\lambda$ in $H$. Let $\nu \in \operatorname{Irr}(K \cap H)$ be the canonical extension of $\lambda$ to $K \cap H$, so that $o(\nu)=p$. If $h \in H$ fixes $\nu$, then $h$ fixes $\lambda$ and therefore $h \in K \cap H$. Hence, by the Clifford correspondence, we have that $\psi=\nu^{H} \in \operatorname{Irr}(H)$. Since $H$ is not cyclic, we have that $K \cap H<H$. Now, if $h \in H-(K \cap H)$, we have that $\nu^{h}=\nu^{r}$ for some integer $r$ with $1<r<p$. Now, $\mathbb{Q}(\psi) \subseteq \mathbb{Q}_{p}$. We claim that $\mathbb{Q}(\psi)$ cannot be $\mathbb{Q}_{p}$. If $\sigma$ is the Galois automorphism fixing $p^{\prime}$-roots of unity and sending $p$-power roots of unity $\xi$ to $\xi^{r}$, then

$$
\psi^{\sigma}=\left(\nu^{r}\right)^{H}=\left(\nu^{h}\right)^{H}=\nu^{H}=\psi,
$$

and this proves the claim.

(3.4) THEOREM. Suppose that $G$ is field equivalent with a cyclic group of order $n$. Then $G$ is cyclic. 
Proof. By hypothesis, we have that

$$
\operatorname{Irr}(G)=\bigcup_{d \mid n} \operatorname{Irr}_{d}(G)
$$

where $\operatorname{Irr}_{d}(G) \cap \operatorname{Irr}_{e}(G)=\emptyset$ if $d \neq e,\left|\operatorname{Irr}_{d}(G)\right|=\varphi(d)$, and if $\psi \in \operatorname{Irr}_{d}(G)$, then $\mathbb{Q}(\psi)=\mathbb{Q}_{d}$. We notice that $G$ has at most two real valued characters. By Lemma (3.1), we have that $P \triangleleft G$, where $P \in \operatorname{Syl}_{2}(G)$. Let $H$ be a 2-complement of $G$.

Suppose that $G$ has odd order. Then $n$ is odd and $G$ satisfies (2.1). If $G$ is nilpotent, then $G$ is cyclic and we are done. By Theorems (2.6) and (2.7), we may assume that $G=N C$, where $C$ is cyclic and $1<N$ is abelian with $(|N|,|C|)=1$. Also, the Sylow subgroups of $N$ are not cyclic and minimal normal subgroups of $G$. Let $p$ be any prime divisor of $|N|$. Now, $G$ has an irreducible character with field of values $\mathbb{Q}_{|C|}$. Hence, $|C|$ divides $n$. Also, by Lemma (2.3), $G$ has an irreducible character with field of values $\mathbb{Q}_{p}$, where $p$ divides $n$. Thus $p|C|$ divides $n$, and $G$ has irreducible characters with field of values $\mathbb{Q}_{p|C|}$. By Theorem (3.2), $G$ has an element $x$ of order $p|C|$. Write $x=u v$, where $u \in N$ has order $p, v$ has order $|C|$ and $u v=v u$. Then $o(v N)=o(v)=|G / N|$, and we deduce that $N\langle v\rangle=G$. Then $u \in \mathbf{Z}(G)$ and $\langle u\rangle$ is a normal subgroup of $G$. Then $\langle u\rangle$ is a Sylow $p$-subgroup of $N$, and this is not possible.

So we may assume that $G$ is of even order. Hence, $n$ is even and $G$ has a unique real valued non-trivial character $\chi$. Let $\delta \in \operatorname{Irr}(P)$ of order 2. By Lemma $(2.3), \delta$ lies under $\chi$, and we deduce that $H$ transitively permutes the nontrivial elements of $\operatorname{Irr}(P / \Phi(P))$. Write $|P / \Phi(P)|=2^{v}$. If $T$ is the stabilizer of $\delta$ in $H$, then $\mathbf{C}_{H}(P) \subseteq T$ and $|H: T|=2^{v}-1$.

Write $n=2^{e} m$, where $m$ is odd. We claim that

$$
\operatorname{Irr}(G / \Phi(P))=\bigcup_{d \mid 2 m} \operatorname{Irr}_{d}(G)
$$

Suppose that $\psi \in \operatorname{Irr}(G)$ has $\Phi(P)$ in its kernel and suppose that $\mathbb{Q}(\psi)=\mathbb{Q}_{f}$ for some $f \mid n$. Now, since the exponent of $G / \Phi(P)$ has 2-part 2, we have that $\mathbb{Q}(\psi) \subseteq \mathbb{Q}_{|G|_{2^{\prime}} \text { and }}$ therefore $f_{2}$ divides 2. Hence, $f$ divides $2 m$. Conversely, suppose that $\psi \in \operatorname{Irr}_{d}(G)$, where $d \mid 2 m$. Then $\mathbb{Q}(\psi)=\mathbb{Q}_{f}$ for some odd number $f$. Let $\mu \in \operatorname{Irr}(P)$ be under $\psi$. Let $\sigma \in \operatorname{Gal}\left(\mathbb{Q}_{|G|} / \mathbb{Q}_{|G|_{2^{\prime}}}\right)$ (which necessarily has 2-power order). Then $\sigma$ fixes $\psi$ and therefore $\mu^{\sigma}=\mu^{x}$ for some $x \in G / P$. Since $o(x)$ is odd, we conclude that $\mu^{\sigma}=\mu$. Hence, $\mu$ has rational values. By Lemma (2.3), we conclude that $\mu$ lies under some rational valued character, which necessarily is $\chi$. Then $\mu$ is $G$-conjugate to $\delta$, and the claim follows.

If $\Phi(P)>1$, arguing by induction, we have that $G / \Phi(P)$ is cyclic. Therefore $P / \Phi(P)$ and $H$ are cyclic. Hence $P$ is cyclic, $G=P \times H$, and therefore $G$ is cyclic. Thus, we may assume that $\Phi(P)=1$. Therefore, $\mathbb{Q}(\psi) \subseteq \mathbb{Q}_{|G|_{2}}$ for all $\psi \in \operatorname{Irr}(G)$. In particular, we have that $n_{2}=2$, since otherwise there would exist $\psi \in \operatorname{Irr}(G)$ such that $\mathbb{Q}(\psi)=\mathbb{Q}_{4}=\mathbb{Q}(i)$, and this is not possible.

Suppose that $P$ is cyclic. Then $|P|=2$ and $G=P \times H$. Then $n=|\operatorname{Irr}(G)|=2|\operatorname{Irr}(H)|$, where $|\operatorname{Irr}(H)|=m$ is odd. Now, for each $d$ dividing $m$, there exist exactly $2 \varphi(d)$ irreducible characters of $G$ with field of valued $\mathbb{Q}_{d}$. If $\chi \in \operatorname{Irr}(G)$, we have that $\chi=1 \times \alpha$ or $\chi=\delta \times \alpha$, for some $\alpha \in \operatorname{Irr}(H)$ and in both cases $\mathbb{Q}(\chi)=\mathbb{Q}(\alpha)$. This easily implies that there are 
exactly $\varphi(d)$ irreducible characters of $H$ with field of values $\mathbb{Q}_{d}$. Hence, $H$ is field equivalent to the cyclic group of $m$ elements, and $H$ is cyclic, by the second paragraph of this proof. Thus $G$ is cyclic in this case. Hence, we may assume that $v \geq 2$.

By Theorem (6.8) of [5], we deduce that $H / \mathbf{C}_{H}(P)$ is a subgroup of $\Gamma$, where $\Gamma$ is as in Lemma (3.3). Now, $H / \mathbf{C}_{H}(P)$ is isomorphic to a quotient of $G$, and therefore all of its irreducible characters have cyclotomic fields of values. By Lemma (3.3), we deduce that $H / \mathbf{C}_{H}(P)$ is cyclic. In particular, $T \triangleleft H$ and we easily have that $T=\mathbf{C}_{H}(P)$.

Notice that the stabilizer of $\delta$ in $G$ is $I=P \mathbf{C}_{H}(P)$. If $\psi \in \operatorname{Irr}(G)$ does not contain $P$ in its kernel, then $\psi$ lies over $\delta$ and therefore $\psi=(\hat{\delta} \alpha)^{G}$, where $\hat{\delta} \in \operatorname{Irr}(I)$ is the canonical extension of $\delta$ to $I$ and $\alpha \in \operatorname{Irr}\left(\mathbf{C}_{H}(P)\right)$. Hence, by using the Clifford correspondence and Corollary (6.17) of [3], we have that

$$
|\operatorname{Irr}(G)|=|\operatorname{Irr}(H)|+\left|\operatorname{Irr}\left(\mathbf{C}_{H}(P)\right)\right| .
$$

Since $H$ is of odd order, by a theorem of Burnside (Problem (3.17) of [3]), we have that

$$
|\operatorname{Irr}(G)| \equiv|H|+\left|\mathbf{C}_{H}(P)\right|=\left|\mathbf{C}_{H}(P)\right|\left(\left|H / \mathbf{C}_{H}(P)\right|+1\right)=2^{v}\left|\mathbf{C}_{H}(P)\right| \bmod 16 .
$$

Hence, we deduce that 4 divides $|\operatorname{Irr}(G)|=n$, and this was not possible.

\section{COPRIME ACTION}

If $X$ and $Y$ are finite groups and $A \subseteq \operatorname{Irr}(X)$ and $B \subseteq \operatorname{Irr}(Y)$, we say that $A$ and $B$ are field equivalent if there exists a bijection $\chi \mapsto \chi^{\prime}$ from $A$ onto $B$ such that $\mathbb{Q}(\chi)=\mathbb{Q}\left(\chi^{\prime}\right)$ for all $\chi \in A$.

(4.1) THEOREM. Suppose that $A$ acts coprimely on $G$ and let $C=\mathbf{C}_{G}(A)$. Then $C$ is cyclic if and only $\operatorname{Irr}_{A}(G)$ is field equivalent with the set of irreducible characters of a cyclic group.

Proof. It is well-known that the Glauberman-Isaacs correspondence * $: \operatorname{Irr}_{A}(G) \rightarrow \operatorname{Irr}(C)$ preserves fields of values. (See Chapter 13 of [3] and Section 10 of [2].) Now, Theorem A applies.

\section{REFERENCES}

[1] J. S. Brodkey, A note on finite groups with an abelian Sylow group. Proc. Amer. Math. Soc. 14 (1963), 132-133.

[2] M. Isaacs, Characters of solvable and symplectic groups, Amer. J. Math. 95 (1973), $594-635$.

[3] M. Isaacs, Character Theory of Finite Groups, Dover, New York, 1994.

[4] S. Iwasaki, On finite groups with exactly two real conjugate classes. Arch. Math. (Basel) 33 (1979/80), no. 6, 512-517.

[5] O. Manz, T. Wolf, Representations of Solvable Groups, Camdridge University Press, 1993.

[6] E. Shult, On finite automorphic algebras. Illinois J. Math. 13 (1969), 625-653. 